\title{
Identification of Blood Vessel Clot Region using Fuzzy C Means Clustering Based Artificial Bee Colony Algorithm
}

\author{
Kottaimalai Ramaraj, Vishnuvarthanan Govindaraj, Pallikonda Rajasekaran Murugan, Yudong Zhang, \\ Shuihua Wang, Arunprasath Thiyagarajan
}

\begin{abstract}
Medical image segmentation results in the multiple fractioning of an input image for a deeper analysis/insight. Localization of objects and detection of boundaries are the coretheme of using segmentation for medical images. It elucidates the process of finding the anatomic structures in medical images. In this paper, we put forth a technique that has Fuzzy C-Means clustering and Artificial Bee Colony (ABC) Optimization has delivered the segmentation of MRA brain image. Artificial Bee Colony $(\mathrm{ABC})$ has been used by many researchers as it is a population-based stochastic approach that has better search-inspace abilities for various optimization problems. The unsupervised clustering FCM has produced candidate outcomes in medical image processing. FCM is mostly preferable for segmenting the soft tissues in brain model, and it provides better output when compared to some of the competitive clustering techniques like KM, EM and KNN. The output of the suggested techniques is verified by using real MRA brain images. The results of Statistical parameters show that our method is notably better compared to other algorithms.
\end{abstract}

Keywords-Magnetic Resonance Angiography (MRA) Image Segmentation, Fuzzy C-Means Clustering (FCM), Artificial Bee Colony optimization (ABC), Blood clot identification.

\section{INTRODUCTION}

The general procedure of the brain's control centers depend on sufficient amount of nutrients and oxygen concluded over the network of left and right mutual carotid arteries and left and right vertebral arteries. The rate of the cerebral blood flow in the adult is usually 750 milliliters per minute. Obstruction to the blood flow in any one of the internal carotid arteries leads to permanent dysfunction of frontal lobes, which might introduce or create the weakness, numbness or paralysis on the side opposite to the obstruction of the artery in our body.

Revised Manuscript Received on December 16, 2019.

Kottaimalai Ramaraj, Department of ICE, Kalasalingam Academy of Research and Education (Kalasalingam University), Srivilliputtur, India. Email: rkottai@gmail.com

Vishnuvarthanan Govindaraj, Department of BME, Kalasalingam Academy of Research and Education (Kalasalingam University) Srivilliputtur, India. Email:gvvarthanan@gmail.com

Pallikonda Rajasekaran Murugan, Department of ECE, Kalasalingam Academy of Research and Education (Kalasalingam University) Srivilliputtur, India. Email: m.p.raja @klu.ac.in

Yudong Zhang, Department of Informatics, University of Leicester, Leicester, LE1 7RH, UK. Email: yudongzhang@ieee.org

Shuihua Wang, Department of Mathematics, University of Leicester, Leicester, LE1 7RH, UK. Email: shuihuawang@ieee.org

Arunprasath Thiyagarajan, Department of ECE, Kalasalingam Academy of Research and Education (Kalasalingam University) Srivilliputtur, India. Email: m.p.raja @klu.ac.in
Blood clots are differently sized clumps of blood that are produced in our body. Blood Clotting is important to prevent excessive bleeding during injury or cut. Although, when a blood clot blocks blood flow to vital areas of our body, it can be harmful, even deadly. Blood clots can occur in our legs and arms, abdomen, heart, brain, lungs and kidneys'. There are two different types of blood clots. The clots that stay in place and don't move called thrombosis and those that break away from the spot where they developed and move to different areas inside your body called embolism. In the brain, the primary symptom of blood clot may be a transient ischemic attack or a stroke. The symptoms of these vary in degrees but include loss of speech and visual impairment, weakness in face, arms or legs, difficulty in understanding others, dizziness, headache and loss of coordination. The morphological imaging test methods like MRI, MRA or CT scan are required to diagnose the presence of a clot and the condition which caused it to appear. Those obtained cross sectional images should be segmented by using image segmentation techniques.

An image refers to the transfer of information and comprises plenty of useful information for further processing and evaluation. Digital image processing supports better to understand and extract the useful information present in the image for various image related applications, and the initial step in this regard is the image segmentation. Image segmentation method renders division of input image into various homogeneous regions, which is a significant primary step in the study of image content. Image segmentation is vivid in applications pertaining to image processing and computer vision. The area of preference/interest could be investigated in a far better way using image segmentation, and this is the basic part of any image analysis process, which facilitates the feature extraction and recognition procedures. For example, instead of analyzing a tumor in a Computed Tomography (CT) volumetric image, image segmentation is used to detect \& isolate tumor from whole image. It helps the physicians to diagnose easily.

\section{LITERATURE SURVEY}

Rajinikanth et al. [1] proposed a hybrid approach that encapsulates pre- and post-processing techniques to visualize MRA images. Initially, Tsallis entropy and Chaotic Firefly Algorithm (CFA) tender tri-level thresholding values that group the common pixels that are contrast enhanced in the 2D MRA images. In post 
processing, deformable snake model of Active Contour (AC) model is applied to extract highly difference portions. Finally, Minkowski distance method helps in assessing the texture property of aneurysm section from MRA and T1contrast enhanced images. The algorithm was unsuccessful in extracting the ROI from T1 modality test images. Phellen et al. [2] suggested a novel automatic segmentation technique that works across 4D MRA images that have the anatomical characteristics and blood flow data, which is unique when compared to other brain imaging modalities. The blood vessels in temporal average intensity projection are better imaged with the influence from the filters named by the authors as of multi-scale vesselness enhancement filters, with further aid from the path operators. Seed based region growing method is used by the authors for segmenting the blood vessels in each frame of the 4D MRA accrued, and utilization of a combined vessel segmentation approach for the same can yield better segmentation outcomes is the verdict made by the authors. Brindha and Nagarajan [3] proposed an algorithm for spinal cord MRI image segmentation using the combination of PBT \& SVM, which gives improved accuracy than ACM and MultiResolution Propagation. Mohammad and Abkenar [4] suggested an algorithm by applying FCM on the brain MRI images. In order to eliminate the noise during the segmentation, $\mathrm{ABC}$ algorithm with Modified Intensity matrix is applied on the images before FCM, and finally better results are obtained. Bogunovic et al. [5] proposed an algorithm to segment aneurysm in the brain arteries $\mathrm{x}$-ray reconstruction angiography with the $3 \mathrm{D}$ and time of flight MRA modality images. The author concluded that the segmented geodesic active regions are better in quality and quantity. Neeraja et al. [6] introduced unsupervised ABC algorithm for tumor segmentation in the brain images. FCM is applied for clustering the images, hence the segmentation quality is improved. Kamalam and karnan [7] implemented a hybrid ABC-FCM algorithm for brain tumor segmentation and achieved better efficiency during segmentation. Emrah et al. [8] introduced a clustering method by using $\mathrm{ABC}$ algorithm and gives improved accuracy compared with other clustering techniques like K-means and PSO. Andres et al. [9] introduced an algorithm to extract the statistical features of brain MRI image. The combinational algorithm, fuzzy clustering and vector quantization gives better solution for the segmentation of partial volume effect. Wang et al. [10] suggested a segmentation technique to analyze the brain vessels from MRA images. By the comparison of two unlike statistical distributions, the threshold segmentation algorithm extracts vessels accurately and efficiently. Wilson and Noble [11] introduced an expectation maximization algorithm on MRA image to extract the 3D vessel data in it accurately. Vishnuvarthanan et al. [12] introduced an algorithm which combines self-organizing maps and fuzzy $\mathrm{k}$ means for image processing. The performance measure values of this algorithm are efficient and have better data handling capacities. Anitha et al. [13] introduced Bacteria Foraging Optimization and modified FKM clustering for brain image segmentation.

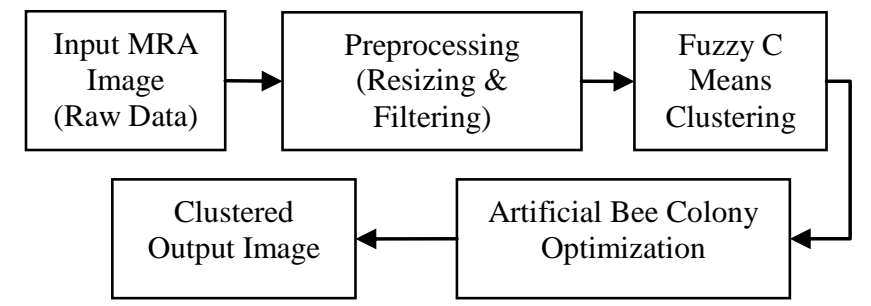

Fig. 1 Proposed Block Diagram

The comparison of the performance measured values of PSO-FCM, modified FKM \& conventional FCM values show that the proposed algorithm produced better values of sensitivity and specificity. Anitha et al. [14] proposed a combination al algorithm having BFO and modified FCM for image segmentation. The proposed algorithm provides better sensitivity and specificity value.

\section{DATASET}

In this paper, the MRA images used are obtained from KGS Advanced MR \& CT Scan, Madurai, Tamilnadu. The dataset has both T1-Weighted (T1-W) and T2-Weighted (T2-W) axial MRA brain images taken from different persons. The suggested techniques were validated on the T1 and $\mathrm{T} 2$ images after preprocessing.

\section{PROPOSED SYSTEM}

The block diagram shown in the Fig.1 represents the proposed algorithm. Contrast limited Adaptive Histogram Equalization (CLAHE) is utilized for preprocessing of MRA images. CLAHE has the ability to evade the noise amplification developing in the homogeneous regions. After resizing and filtering the image by preprocessing, FCM and $\mathrm{ABC}$ are applied for segmentation.

\section{A. Fuzzy C-Means Clustering:}

Fuzzy clustering is one among the noteworthy unsupervised learning methods also known as soft clustering. Each element in this cluster is allotted a membership value which is equivalent to degree of function in that cluster. Fuzzy K-means and k-medoids are called as hard clustering. For the fuzzy clustering, location of points near to the center of a cluster, may be in the cluster to a higher degree than points in the edge of a cluster. Each element in a cluster has a numerical value between 0 and 1 . FCM is the extensively used fuzzy clustering method among different fuzzy algorithms proposed by Bezdek. The cluster centroid is considered as the average of all pixels respective to the degree of cluster belongs. The cluster is formed by grouping all the nearby associated similar elements. It allocates a membership value to data point, subject to the resemblance of the data point to a specific class comparative to the remaining ones.

FCM looks like k-means and its theme is to minimize the objective function by segmenting the image in to clusters as follow: 
$\sum_{j=1}^{K} \sum_{x_{1} \in C_{j}} u_{i j}^{m}\left(x_{i}-\mu_{j}\right)^{2}$

Where,

$=$ membership value of $\mathrm{j}^{\text {th }}$ data in $\mathrm{i}^{\text {th }}$ cluster

$=$ centroid of the cluster $\mathrm{j}$

$\mathrm{m}=$ index of fuzziness

$$
X=\text { a data in size }
$$

$=$ dimension of each vector

=number of pixels in an image

=Euclidean norm

The objective function of the cluster is reduced only when the data points which are far away from the centroid are allotted as low membership values and the data points near to the centroid are allocated as high membership values.

The term is described as follow:

$$
u_{i j}^{m}=\frac{1}{\sum_{i=1}^{k}\left(\frac{\left|x_{i}-c_{j}\right|}{\left|x_{i}-c_{k}\right|}\right)^{\frac{2}{m-1}}}
$$

The membership degree, is inversely correlated to the distance from $\mathrm{x}$ to the cluster center.

$C_{j}=\frac{\sum_{x \in C j} u_{i j}^{m} x}{\sum_{x \in C j^{j}} u_{i j}^{m}}$

Where,

$=$ centroid of the cluster $\mathrm{j}$

The iteration of the FCM proceeds with an ultimatum of reaching two main goals, one to associate the membership value and the classes for the pursuance of clustering, second is to assign higher membership value for the nearest data points, through which the segmentation of the data can be achieved.

The basic algorithm steps for FCM are,

1. Initialize random centroid to all clusters.

2. Measuring the distance between the individual point and cluster center.

3. Re-calculate the membership function, depending upon the distance between the individual point and cluster center.

4. Re-calculate the centroid, as per the new membership function.

5. If the variation between the new centroid and the original one is less than threshold value, then the algorithm halts, otherwise it remains till this condition is true.

\section{B. Artificial Bee Colony Optmization:}

Artificial Bee Colony (ABC) by Dervis Karaboga is an optimization technique which mimics the intellectual characteristics of honey bees. In ABC, the parameters are controlled by maximum number of cycles and colony size which is similar to Differential Evolution and PSO. ABC proposes a population-based search method, where the entities are position of food. The bee's desire is to determine high nectar. The bees fly ubiquitously in several directions. The onlooker bees and the employed bees find the high nectar food sources as per their expertise and alter their food positions in the memory. But scout bees fly and select the food origin arbitrarily except any expertise. They memorize the high nectar food source position and forget the earlier one in its memory. ABC is a better algorithm for balancing both exploration and exploitation in the search process.

The ABC algorithm contains following stages:

- Initialization Stage:

The primary nectar sources for bees are randomly created using

$x_{m}=l_{i}+\operatorname{rand}(0,1) *\left(u_{i}-l_{i}\right)$

Where,

$l_{i}$ and $u_{i}=$ lower $\&$ upper bound of objective function $\operatorname{rand}(0,1)=$ arbitrary value between the range $0 \& 1$.

- Employed bee Stage:

The surrounding or nearby food source $v_{m i}$ is

$v_{m i}=x_{m i}+\varphi_{m i}\left(x_{m i}-x_{k i}\right)$

Where,

$i=$ index parameter,

$x_{k}=$ food source selected arbitrarily,

$\varphi_{m i}=$ arbitrary number between the range $[-1,1]$. The fitness function is determined as

fit $_{m}\left(x_{m}\right)=1+\left\|f_{m}\left(x_{m}\right)\right\|_{b} f_{m}\left(x_{m}\right)<0$

Where,

$f_{m}\left(x_{m}\right)=$ objective function of $x_{m}$.

- Onlooker Bee Stage:

The size of the food source is measured by its profitability $P_{m}$ as

$$
P_{m}=\frac{f i t_{m}\left(x_{m}\right)}{\sum_{m=1}^{S N} f i t_{m}\left(x_{m}\right)}
$$

Where,

fit $t_{m}\left(x_{m}\right)=$ fitness of $x_{m}$

As per the below expression the onlooker bees looking for food sources in their nearby regions

$v_{m i}=x_{m i}+\varphi_{m i}\left(x_{m i}-x_{k i}\right)$

- Scout Stage:

The scout bees arbitrarily explored the new food sources.

The new food source with high nectar is

$$
x_{\mathrm{m}}=l_{\mathrm{i}}+\operatorname{rand}(0,1) *
$$

\section{IMPLEMENTATION}

Initially, the obtained dataset images should be ROI extracted and then given to clustering. The ROI extracted image is given as an input image in our proposed algorithm. The input image is resized and then filtered in the preprocessing stage. After that, FCM is applied on those images for better clustering output. Then Artificial Bee Colony optimization is employed on the FCM clustered image. Finally, by using the proposed algorithm, the blood clot area in the brain MRA images is extracted for better visibility. 


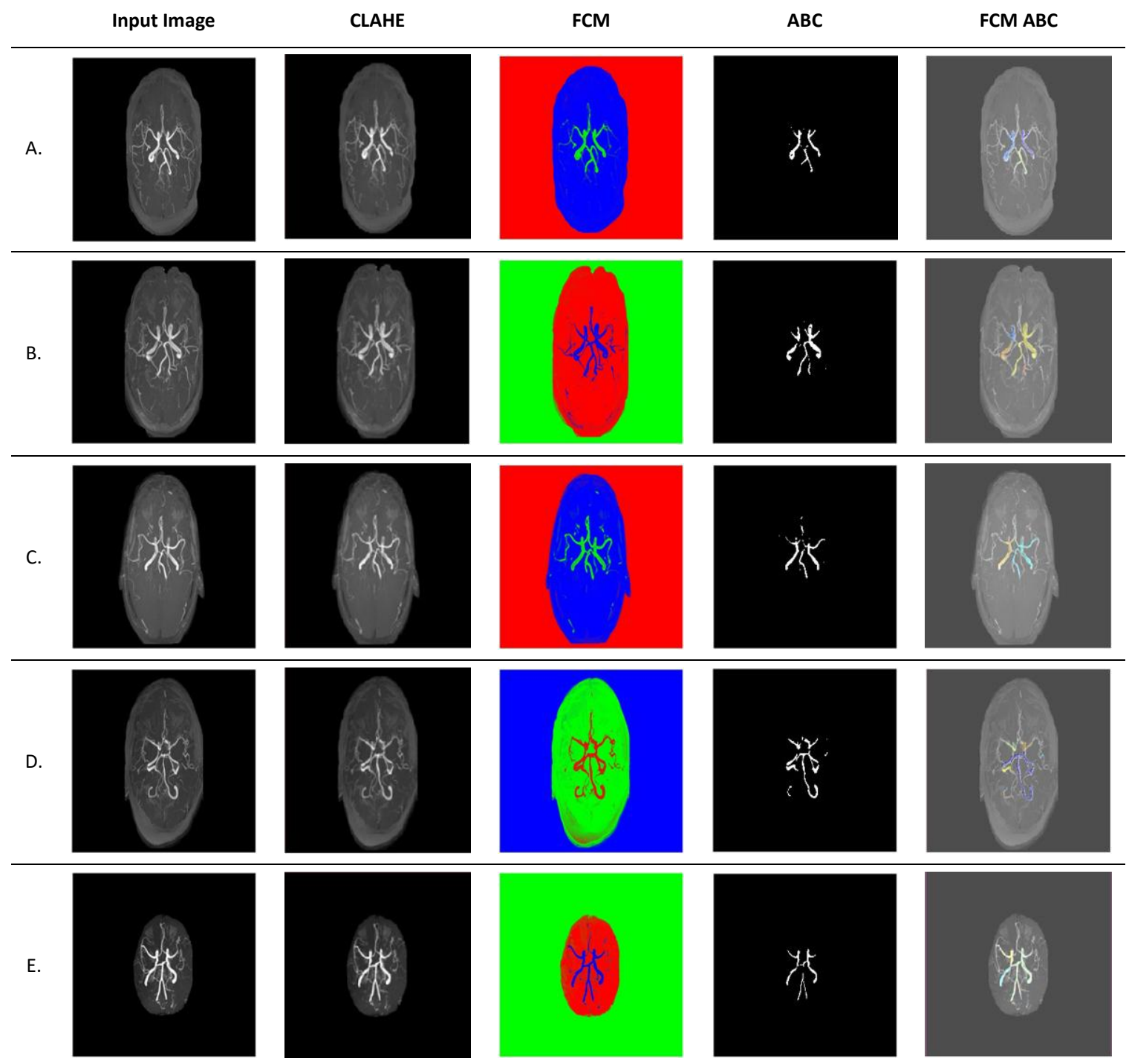

Fig. 2 Segmented results of dataset

\section{RESULTS AND DISCUSSION}

The efficiency of FCM algorithm is proved by taking some complicated cases which are very difficult to diagnose during clinical actions. From Fig. 2. (A) to (E), FCM-ABC results empower the FCM results, and in that,the brain blood clots are clearly identifed. Also, Fig. 2. (A), (B), (C) and (E) represent the excellence of the algorithm, which the blood clot portions are identified with high level of accuracy.

Table I. Performance Analysis Using Comparison Metrics

\begin{tabular}{|c|c|c|c|c|}
\hline \multirow{2}{*}{ Patient } & \multicolumn{4}{|c|}{ Performance Measures } \\
\cline { 2 - 5 } & MSE & $\begin{array}{c}\text { PSNR in } \\
\mathrm{dB}\end{array}$ & TC in \% & DOI in \% \\
\hline $\mathrm{A}$ & 4.5218 & 41.5700 & 53.22 & 69.47 \\
\hline $\mathrm{B}$ & 2.0438 & 45.0265 & 51.99 & 68.41 \\
\hline $\mathrm{C}$ & 4.4598 & 41.6376 & 51.99 & 68.41 \\
\hline $\mathrm{D}$ & 4.5696 & 41.5320 & 52.19 & 68.59 \\
\hline E & 4.7615 & 41.3533 & 74.44 & 85.35 \\
\hline
\end{tabular}

Fig. 3 Graphical representation of the performance analysis metrics

Table I gives the benchmark metric values for the proposed algorithm such as, Peak Signal to Noise Ratio (PSNR), Mean Squared Error (MSE), Dice Overlap Index (DOI) and Jaccard Tanimoto Cofficient (TC).

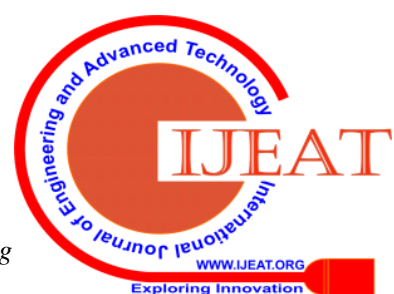




\section{CONCLUSION}

The recommended FCM-ABC algorithm processes 42 MRA brain slices with various blood clot complicacies with variance in noise levels. The FCM-ABC algorithm responses well to intensity inhomogeneity, noise and poor image contrast, which demonstrates its over-all segmentation property. These are clearly illustrated in Fig. 2. Further reduction of MSE values and subsequent increase in PSNR values, with improvement in TC and DOI values are considered to be the furture scope of this research work.

\section{ACKNOWLEDGMENT}

We thank the Department of Instrumentation and Control Engineering of Kalasalingam University, (Kalasalingam Academy of Research and Education), Tamil Nadu, India for permitting to use the computational facilities available in Biomedical Laboratory which was setup with the support of the Department of Science and Technology (DST), New Delhi under FIST Program. Also we thank Dr.K.G.Srinivasan, MDRD, Consultant Radiologist and Dr.K.P.Usha Nandhini, DNB, KGS Advanced MR \& CT Scan - Madurai, Tamilnadu, India, behind the research with the patient information.

\section{REFERENCES}

[1] V.Rajinikanth, Nilanjan Dey, Suresh Chandra Satapathy and Amira S Ashour, "An approach to examine magnetic resonance angiography based on Tsallis entropy and deformable snake model," Future Generation Computer Systems (2018).

[2] R.Phellan, T.Lindner, M.Helle, A.X.Falcao and N.D.Forkert, "Automatic temporal segmentation of vessels of the brain using 4D ASL MRA images," IEEE Transactions on Biomedical Engineering, 0018-9294 (c) 2017 IEEE, DOI 10.1109/TBME.2017.2759730.

[3] D.Brindha and N.Nagarajan, "An efficient automatic segmentation of spinal cord in MRI images using interactive random walker (RW) with artificial bee colony (ABC) algorithm" Springer Science+Business Media, LLC, part of Springer Nature 2018, DOI: g/10.1007/s11042-018-6331-8.

[4] Mohammad Shokouhifar and Gholamhasan, "An artificial bee colony optimization for MRI fuzzy segmentation of brain tissue", 2011 International Conference on Management and Artificial Intelligence.

[5] Hrvoje Bogunovic, Jose Maria Pozo, Maria Cruz Villa Uriol, Charles B.L.M. Majoie, Rene van den Berg, Hugo A.F. Gratama van Andel, Juan M.Macho, Jodi Balsco, Luis San Roman and Alejandro F.Frangi, "Automated segmentation of cerebral vasculature with aneurysms 3DRA and TOF-MRA using geodesic active regions: An evaluation study" Medical Physics, Vol.38, No.1, January 2011.

[6] Neeraja R Menon, M.Karnan and R.Sivakumar, "Brain tumor segmentationin MRI image using unsupervised artificial bee colony and FCM clustering" International Journal of Computer Science and Management Research, vol. 2, Issue 5 May 2013, ISSN 2278-733X.

[7] Kamalam Balasubramani and Karnan Marcus, "Artificial bee colony algorithm to improve brain MRI image segmentation," Internationa Journal on Computer Science and Engineering, Vol. 5 No. 01 Jan 2013, ISSN : 0975-3397.

[8] Emrah Hancer, Celal Ozturk and Dervis Karaboga, "Artificial bee colony based image clustering method,"WCCI 2012 IEEE World Congress on Computational Intelligence, 978-1-4673-15098/12/\$31.00 @2012 IEEE.

[9] Andres Ortiz, Antonio A. Palacio, Juan M. Gorriz, Javier Ramirez and Diego Salas Gonzalez, "Segmentation of brain MRI using SOMFCM-based method and 3D ststistical descriptors," Computational and mathematical methods in medicine, Volume 2013, Article ID 638563, DOI: $10.1155 / 2013 / 638563$.

[10] Rui Wang, Chao Li, Jie Wang, Xiaoer Wei, Yuehua Li, Yuemin zhu and Su Zhang. "Threshold segmentation algorithm for automatic extraction of cerebral vesssels from brain magnetic resonance

angiography images," Journal of Neuroscience Methods Elseiver 2014.

[11] D. L. Wilson and J. A. Noble, "An adaptive segmentation algorithm for time of flight MRA data," IEEE transactions on medical imaging, vol. 18 , no. 10 , october 1999

[12] G. Vishnuvarthanan, M. P. Rajasekaran, P. Subbaraj and V. Anitha, "An unsupervised learning method with a clustering approach for tumor identification and tissue segmentation in magnetic resonance brain images," Applied Soft Computing, vol. 38, pp.190-212, 2016.

[13] V. Anitha, M. P. Rajasekaran, G. Vishnuvathanan,Yudong Zhang and T. Arunprasath, "An automated hybrid approach using clustering and nature inspired optimization technique for improved tumor and tissue segmentation in magnetic resonance brain images," Applied Soft Computing,vol. 57, pp.399 - 426, 2017.

[14] V. Anitha, M. P. Rajasekaran, G. Vishnuvathanan,Yudong Zhang and T. Arunprasath, "Development of a combinational framework to concurrently perform tissue segmentation and tumor identification in T1-W, T2-W, FLAIR and MPR Type magnetic resonance brain images," Expert Systems with Applications,vol. 95, pp.280 - 311, 2018 .

\section{AUTHORS PROFILE}

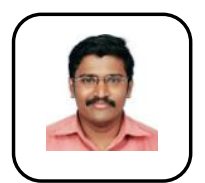

R.Kottaimalai working as an Assistant Professor in the Department of Electronics and Communication Engineering at Sree Sowdambika College of Engineering, Aruppukottai, Tamilnadu, India. He is graduated in Instrumentation and Control Engineering at Arulmigu Kalasalingam College of Engineering, Krishnankoil in 2007. He secured M.E. Degree in Control and Instrumentation from Kalasalingam University in 2013. He is in teaching profession for more than 6 years. He has presented number of papers in National and International Symposiums and Conferences. His main areas of interest include Digital Signal Processing, Image Processing and Control System. He worked as a New Product Development and Testing Engineer in Automobile ancillaries manufacturing companies like Vibromech Engineers and Services Limited and Himu Accessories Private Limited in Chennai for 4 years. He is a life member of ISTE.

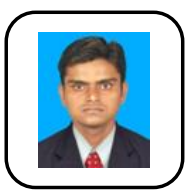

Dr.G.Vishnuvarthanan, born in 1986, has research stints in the avenues of medical image processing and artificial intelligence. He was awarded $\mathrm{PhD}$ in the year 2015 and bachelor's degree in Instrumentation and Control Engineering by 2007, and Master's Degree in VLSI by 2009. He has more than ten years of teaching and research experience and has his affiliation as Associate Professor with the Department of Biomedical Engineering of School of Bio and Chemical Sciences in the Kalasalingam Academy of Research and Education, Tamilnadu, India. He has more than 17 publications in SCI journals and 20 International Publications which have been indexed in SCOPUS database. He is a life member of ISTE.

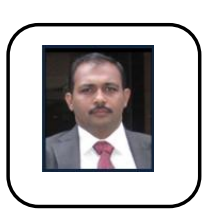

M.Pallikonda Rajasekaran, born in Srivilliputhur, Virudhunagar District of Tamil Nadu in 1980, he had his schooling in the same town and graduated in Electronics and Instrumentation Engineering in 2001 from Shanmugha College of Engineering, Thanjavur and completed his M.Tech. degree in 2002 with second Rank in SASTRA University. He pursued his doctoral programme in Anna University, Chennai. Starting as a Lecturer in 2003, he became Asst. Professor in 2008, Associate Professor in 2009 and Professor in 2012 in Kalasalingam Academy of Research and Education. He had a deep involvement in Bio-signal Processing research. His work majorly focuses on the Image Segmentation for identification of brain tumour and image reconstruction and compression using medical images for diagnosis Over 150 B.Tech students, 75 M.Tech students, and 8 Doctorates stand testimony for his productivity in Image Processing, Wireless Sensor Networks, and Biomedical Instrumentation research. He has so far published more than 50 papers in national and international journals and conferences. He is a Fellow of Indian Society For Technical Education (ISTE), Institute of Electrical and Electronics Engineers (IEEE), AsiaPacific Chemical, Biological \& Environmental Engineering Society (APCBEES), Institution of Engineers (India)(IE), International Association of Engineers (IAENG) and International Association of Computer Science and Information Technology (IACSIT). 


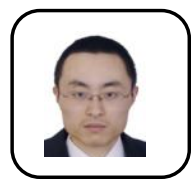

Prof. Yu-Dong Zhang received his $\mathrm{PhD}$ degree from Southeast University in 2010. He worked as a Post-doc from 2010 to 2012 in Columbia University, USA, and as an assistant research scientist from 2012 to 2013 at Research Foundation of Mental Hygiene (RFMH), USA. He served as a full professor from 2013 to 2017 in Nanjing Normal University, where he was the director and founder of Advanced Medical Image Processing Group in NJNU. Now he serves as Professor in Department of Informatics, University of Leicester, UK. He was included in "Most Cited Chinese researchers (Computer Science)" by Elsevier from 2014 to 2018. He was the 2019 recipient of "Highly Cited Researcher" by Web of Science. He won "Emerald Citation of Excellence 2017" and "MDPI Top 10 Most Cited Papers 2015". He was included in "Top Scientist" in Guide2Research. He published over 160 papers, including 16 "ESI Highly Cited Papers", and 2 "ESI Hot Papers". His citation reached 10096 in Google Scholar, and 5362 in Web of Science. He is the fellow of IET (FIET), and the senior members of IEEE and ACM. He is the editor of Scientific Reports, IEEE Transactions on Circuits and Systems for Video Technology, etc. He served as the (leading) guest editor of Information Fusion, Neural Networks, IEEE Transactions on Intelligent Transportation Systems, etc. He has conducted many successful industrial projects and academic grants from NSFC, NIH, Royal Society, and British Council.

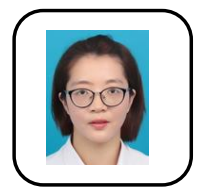

Dr.Shuihua Wang received her Ph.D. degree from Nanjing University. She worked as an assistant professor in Nanjing Normal University from 20132018 and also served in Loughborough University from 2018-2019. She received the B.S. degree from Southeast University, Nanjing, China, in 2008, the M.S degree from The City University of New York, New York, NY, USA, in 2012. Now, she works as a Research Associate in University of Leicester. Her research interests include biomedical image processing and computeraided diagnosis. She published more than $100 \mathrm{SCI}$ indexed papers, 12 were included as "ESI highly cited paper" and 2 were included as "ESI Hot Paper".

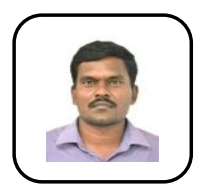

Dr.T.Arunprasath is an Associate professor in the Department Biomedical Engineering at Kalasalingam Academy of Research and Education. He received his $\mathrm{Ph} . \mathrm{D}$. in Electronics and Communication Engineering from Kalasalingam University, Krishnankoil in 2015, his M.E. in Applied Electronics from Anna University in 2009 (Mohamed Sathak Engineering College) and his B.E. in Electrical and Electronics Engineering from Anna University (Syed Ammal Engineering College) in 2006. His research interests include biomedical instrumentation, image processing, image segmentation cloud computing, image segmentation. He has published 16 technical journals and 20 technical papers in refereed conferences in these areas. He is a life member of ISTE. 